\title{
What influences the home range size of free-roaming domestic dogs?
}

\author{
S. DÜRR ${ }^{1}$, N. K. DHAND ${ }^{2}$, C. BOMBARA ${ }^{2}$, S. MOLLOY ${ }^{2}$ AND M. P. WARD ${ }^{2}$ \\ ${ }^{1}$ Veterinary Public Health Institute, Vetsuisse Faculty, University of Bern, Switzerland \\ ${ }^{2}$ Sydney School of Veterinary Science, The University of Sydney, Australia
}

Received 17 October 2016; Final revision 15 December 2016; Accepted 17 January 2017; first published online 16 February 2017

\section{SUMMARY}

In many regions of the world domestic dogs are free roaming and live in close relationship with humans. These free-roaming domestic dogs (FRDD) can cause public health problems such as dog bites and transmission of infectious diseases. To effectively control diseases transmitted by FRDD, knowledge on the dogs' behaviour is required. To identify predictors of home range (HR) size, we collected global positioning system data from 135 FRDD living in eight Aboriginal and Torres Strait Islander communities in Northern Australia. The core HR size ranged from 0.17 to 2.33 ha and the extended HR size from 0.86 to 40.46 ha. Using a linear mixed effect model with a Restricted Maximum Likelihood approach, the dog's sex and reproductive status were identified as predictors of roaming. Non-castrated males had the largest HRs, followed by neutered females. Also, FRDDs were found to roam further during the prethan the post-wet season. These findings have implications for infectious disease spread.

Identification of risk groups for disease spread within a population allows for more targeted disease response and surveillance. Further investigation of predictors of roaming in other FRDD populations worldwide would increase the external validity of such studies.

Key words: Canine, diseases spread, global positioning system, predictors, REML mixed effect model.

\section{INTRODUCTION}

Domestic dogs (Canis familiaris) are among the oldest and most widespread domesticated animals [1]. In many regions dogs live continuously (or partly) in free-roaming populations; this includes dogs that are owned by individual persons, families or neighbourhoods, or those which are ownerless [2]. These freeroaming domestic dogs (FRDD) often cause public and animal health and welfare problems, both for

\footnotetext{
* Author for correspondence: S. Dürr, Vetsuisse Faculty, Veterinary Public Health Institute, University of Bern, Schwarzenburgstrasse 155, CH-3097 Liebefeld, Switzerland. (Email: salome.duerr@vetsuisse.unibe.ch)
}

domestic animals and wildlife [3]. These problems include dog bite injuries, pollution, noise and the spread of both zoonotic and non-zoonotic diseases. The former includes rabies, echinococcosis (hydatidosis), visceral leishmaniasis, mange (Sarcoptes scabiei) and giardiasis [4-9]; the latter haemoparasites (Anaplasma spp., Babesia spp., Ehrlichia spp.), heartworm (Dirofilaria immitis) and hookworm (Ancylostoma caninum) [10-12].

It was concluded 15 years ago that published studies on FRDD ecology are rare [2]; little has changed since then. Published studies are mainly from regions with endemic canine rabies [2], because rabies is the disease with the greatest public health concern in relation to FRDD populations [13], killing an estimated 
59000 people per year worldwide [14]. In relation to control of canine diseases such as rabies or hydatidosis, knowledge of FRDD population size, demography and dynamics are crucial [15-22]. Besides knowledge of the demography of FRDD populations, the ability to access dogs (which depends on the relationship between humans and dogs) is essential for the planning of control strategies such as mass vaccination or anti-parasitic treatments. It has been demonstrated in selected communities in Indonesia and South Africa that human attitudes and needs strongly influence dog population size and movements of dogs between regions and therefore, the success of rabies control actions [19].

The roaming behaviour of FRDD - including movements and social patterns - is another factor that informs disease control efforts, because diseases spread with the movements of their hosts. Knowledge of home range (HR) sizes and shapes of both the 'typical' and far-roaming dogs is required to estimate the extent and speed of spread of disease outbreaks. This knowledge can also be used to identify sub-populations capable of transmitting diseases over a larger area and are therefore dogs that need to be targeted for control actions. This topic has rarely been explored and studies only cover restricted areas and mostly include a limited number $(\leqslant 17)$ of dogs [23-27]. Only a few studies with larger sample sizes have been published [28-30]. The direct connection between roaming behaviour and disease spread by dogs was investigated for echinococcosis in Kyrgyzstan [29] and Tibet [30], where individual dog global positioning system (GPS) and faecal worm burden data were collected.

Several factors may influence the roaming behaviour of FRDD, including intrinsic (such as sex, reproductive status, age, breed), human associated (such as dog transport, use of the dogs, feeding practices), population associated (dog density, social structure of the population) and extrinsic (season, climate, environment) ones. Sex and reproductive status have already been explored and results published, but with some contradictory results [23, 28-30] regarding HR sizes. In rural and remote regions where the majority of dogs are free roaming - genetic exchange with wildlife may occur when conspecifics are present. This has been demonstrated in Australia where cross-breeds between domestic dogs and dingoes were frequently found [31]. However, no influence of the dingo ancestry on HR has been identified so far [32].
In the current study, we use previously published $[28,32,33]$ and new data on HR sizes of FRDD living in Australian Aboriginal and Torres Strait Islander communities to identify potential predictors of HR size. Repeated data collected from 135 dogs produced 215 observations that were included in this study. The aims of the study were to identify both intrinsic and extrinsic predictors of the roaming of FRDD. Additional possible predictors not investigated here and the relevance of the results for disease spread and control in FRDD populations are discussed.

\section{METHODS}

\section{Dataset}

Data on HR sizes of 135 dogs were analysed. The dogs lived in Aboriginal and Torres Strait Islander communities in three regions in the Northern Territory and Queensland, Australia (Fig. 1). In the first region, Seisia, New Mapoon, Bamaga, Umagico and Injinoo are located in close proximity $(2-4 \mathrm{~km}$ from each other) in a remote area in the Northern Peninsula Area (NPA, $10.883^{\circ} \mathrm{S}, 142.383^{\circ} \mathrm{E}$ ), the most northern point of mainland Australia. In the most recent census (2011, http://www.censusdata.abs. gov.au) 2272 people were reported to reside in the NPA, and in a 2009 dog survey 473 dogs were registered in the five communities [28]. The second region is Elcho Island $\left(11.917^{\circ} \mathrm{S}, 135.750^{\circ} \mathrm{E}\right)$ in the Northern Territory where Galiwin'ku, a single large community, is located. In 2011 (http://www.censusdata.abs.gov.au) 2124 residents were reported to live in Galiwin'ku and the dog population was estimated to be 350 [28]. All of these six communities are coastal, located within $3.5 \mathrm{~km}$ of the sea. Seisia has a ferry wharf with frequent connections to and from Thursday Island in the Torres Strait. The third region is located further inland in the Northern Territory around Katherine $\left(14 \cdot 452^{\circ} \mathrm{S}, 132 \cdot 272^{\circ} \mathrm{E}\right)$ with two communities (Katherine I and Katherine II) integrated into the study. These communities are situated 270 and $150 \mathrm{~km}$ from the closest coastline (Gulf of Carpentaria) and have several neighbouring communities. A total of 953 residents and an estimated 266 dogs live in these two communities (Burleigh et al. [34]; http://www.censusdata.abs.gov.au). The dog density in the eight communities ranged between 57 and $450 \mathrm{dogs} / \mathrm{km}^{2}$. Dogs are usually owned but typically free roaming day and night, although some are kept inside the yard or chained up temporary. 


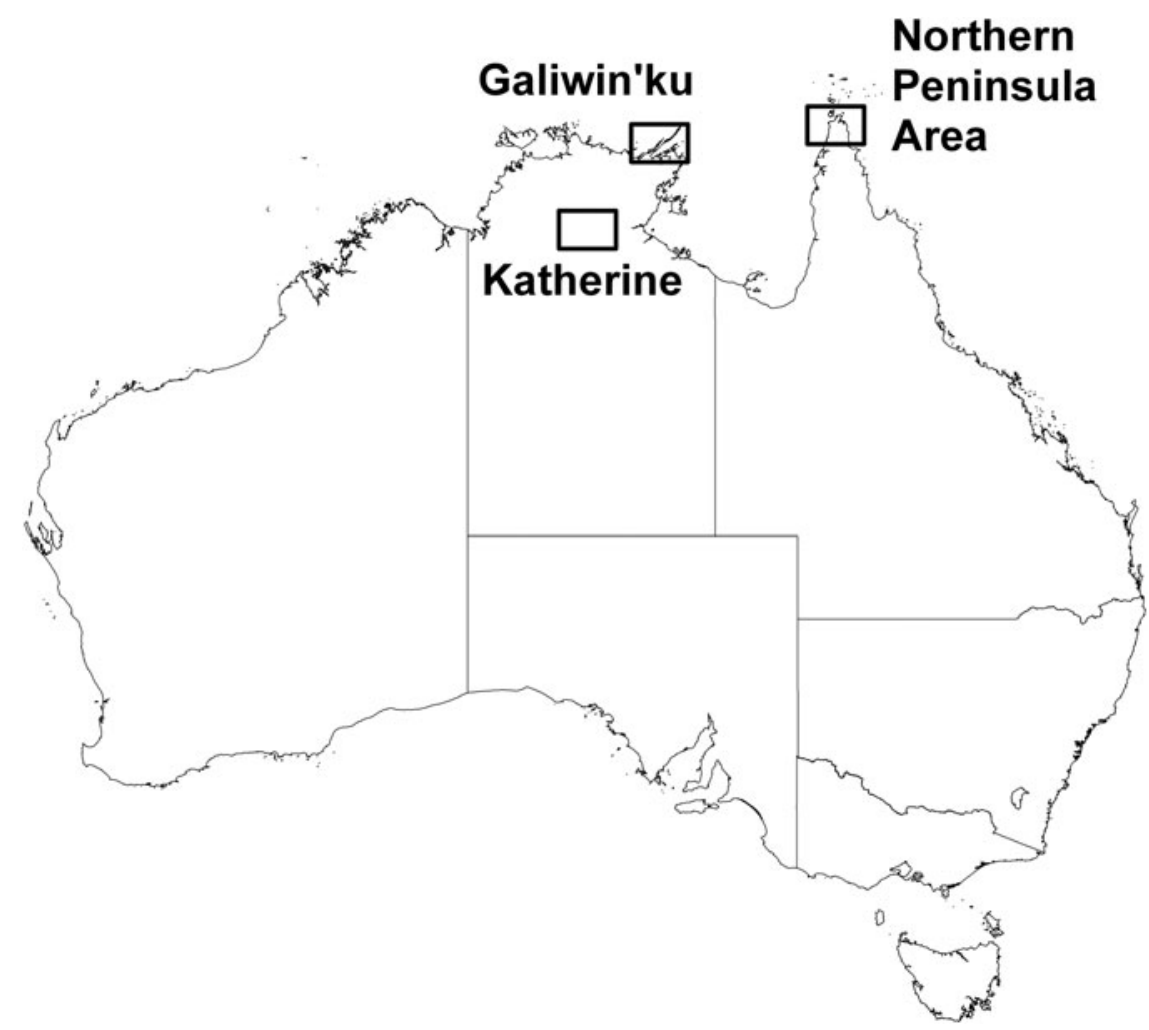

Fig. 1. Map of northern part of Australia with the study regions. The Northern Peninsula Area is located in Queensland, Galiwin'ku and Katherine A and B in the Northern Territory.

GPS units (CatLog, www.mr-lee.com) were attached to opportunistically selected dogs using common dog collars, as previously described [28, 33]. The accuracy of the GPS units was assessed in previous studies in Northern Australia [28] and in a rural landscape in France [35]. The mean location errors were found to be acceptable with 18.3 and $15.4 \mathrm{~m}$, respectively. The dogs were collared between one and four times, resulting in a total of 268 collars used in the field. Data collection time point A was in September-November 2013, time point B in April 2014, time point C in September-October 2014 and time point D in March-June 2015. If possible, the same dogs were re-collared at the consecutive time point; however, when the dog was absent (died, moved to outstations, whereabouts unknown) or the owner was absent, it was replaced by another dog (and where possible, from the same household). The recording interval of GPS fixes was set at $1 \mathrm{~min}$. GPS collars were left with the dogs for a period of 2-16 days. After retrieval of the collars, data were downloaded and cleaned by excluding outliers and obvious car trips [28].

The study was approved by the Animal Ethics Committee (\# N00/7-2013/2/6015) and the Human Ethics
Committee (\# 2013/757) of the University of Sydney and was supported by the NPA Regional Council and the Community Advisory Board of Galiwin'ku.

\section{The outcome variables}

The HR size of the study dogs was calculated using the Biased Random Bridge (BRB) method as described in detail by Benhamou [36]. In previous research in the study areas, this was found to be the most suitable method for estimating HR size [28]. Briefly, the BRB method uses a probability density function over discrete-step movements from one GPS fix to the next. The density kernel of the movements was calculated and the areas using two isopleth levels of the kernel (50\% and 95\%) were extracted. The 'adehabitatLT' package in the software R (http://cran. r-project.org) was used for the analysis. The HR50 and HR95 were defined as the core and extended $\mathrm{HR}$, respectively, of the study dogs and were used as the outcome variables of interest. The distributions of outcome variables were right (positively) skewed; these were $\log$ transformed to meet the assumption of normality and homoscedasticity. 


\section{The explanatory variables tested as predictors}

Ten variables were assessed as predictors of HR sizes, and were either intrinsic (dog individual) or extrinsic predictors (Table 2). Intrinsic predictors included (a) sex (male or female), recorded based on observation; (b) reproductive status (entire or neutered), recorded based on observation for males and the statement of the owners or presence of a surgery scar for females; (c) age (young ( $0-1$ year old); adult ( $>1$ to $<6$ years old); or old ( $>6$ years old)), based on the owner's statements or on observation; (d) breed, based on observation where the common community cross breeds were defined as 'community dog' and known breeds were divided into small, medium and large breeds; (e) whether or not the dog was used for (mostly pig) hunting, based on the statement of the owners; and (f) and (g), two measures of dog genetics on continuous and binary scales: the percentage of dingo DNA in community dogs (f) and whether this percentage exceeds $10 \%$, which was defined as hybrids (g) [31]. The extrinsic predictors included (h) the region and (i) the community in which the dog was living and (j) the season when data collection was conducted. The season was divided into pre-wet (data collection time points A and $\mathrm{C}$ ) and post-wet (data collection time points $\mathrm{B}$ and D) season. The main rainy season occurs between November and March. Associations between explanatory variables were tested using $\chi^{2}$ tests.

\section{Analysis of predictors of roaming}

A linear mixed effect model with the Residual or Restricted Maximum Likelihood (REML) approach was used to analyse data. The analyses were performed in R software using the packages 'nlme' (lme function). The REML method was selected because the data were clustered (repeated measures of individual dogs) and the design was unbalanced because the number of observations per dog varied.

In the first step, all predictors (Table 2) and the region and community were tested in univariable models, for both HR50 and HR95 (separately). The variable 'Dog_ID', representing the identification code for each dog, was included as a random effect to account for multiple observations per dog. In total, 10 predictors were tested in univariable models and predictors with marginal (overall) $P$ values $<0 \cdot 3$ were selected for the next step.

In a second step, all predictors selected following screening were included in the multivariate mixed effect models and, using a backwards elimination approach, the predictors with highest $P$ values were stepwise excluded. The Akaike's Information Criterion (AIC) was noted at each step when a variable was excluded to ensure that the more parsimonious model with the smallest AIC was selected. The predictors region and community were included separately in two distinct, otherwise identical, models. Because of the biological association of sex and reproductive status, both predictors including their first-order interaction were further considered, in case the univariable analysis resulted in a $P$ value of $<0 \cdot 3$. The final model included only significant predictors $(P<0 \cdot 05)$. After this, the biologically plausible predictors were tested as confounders with the exception of the two genetic variables and hunting, because the inclusion of these variables would substantially reduce the number of observations in the dataset (Table 2). When a confounder was detected, i.e. the coefficient values of the fixed effects changed $>20 \%$, this predictor was retained in the model.

Finally, model assumptions were investigated by checking for (a) normal distribution of the residuals using histograms and normal Q-Q plots and (b) homoscedasticity by visualising the fitted model values $v s$. standardised residuals.

\section{RESULTS}

\section{Dataset and HR sizes}

In total, 215 sets of GPS data from 135 dogs were available for HR size estimation and predictor analysis, a success rate (usable GPS datasets as a proportion of the total number of collars deployed in the field) of $80 \cdot 2 \%(215 / 268)$. Reasons for missing suitable GPS data included loss and failure of the GPS units. For most $(63 \%)$ of the dogs data from only one time point were available; data from two, three and four time points were available from $19 \%, 15 \%$ and $3 \%$ of dogs, respectively (Table 1). Information on predictors were available from 56 ( $41 \%$ of the study population) to $135(100 \%)$ dogs, depending on the predictor (Table 2). Significantly more females $(35 / 52=67 \%)$ were neutered than males $(30 / 70=43 \%)\left(\chi^{2}=6 \cdot 22\right.$, $P=0.013)$. Neutered dogs were significantly more often found in Galiwin'ku $(35 / 39=90 \%)$ than in the NPA $(18 / 57=32 \%)$ and inland Northern Territory $(12 / 26=46 \%) \quad\left(\chi^{2}=31 \cdot 1, P<0 \cdot 001\right)$. Genetics were only assessed in the NPA and Galiwin'ku and significantly more hybrid dogs were found in Galiwin'ku $(11 / 25=44 \%)$ than in the NPA $(1 / 31=3 \%)\left(\chi^{2}=11 \cdot 4\right.$, 
Table 1. Number of free-roaming domestic dogs per community and data collection time point used in a GPS collar study in three regions in Northern Australia between September 2013 and June 2015

\begin{tabular}{|c|c|c|c|c|c|c|c|c|c|}
\hline \multirow[b]{2}{*}{$\begin{array}{l}\text { Time point of data } \\
\text { collection }\end{array}$} & \multicolumn{5}{|c|}{ Northern Peninsula Area } & \multirow{2}{*}{$\begin{array}{l}\text { Elcho } \\
\text { Island } \\
\text { Galiwin'ku }\end{array}$} & \multicolumn{2}{|c|}{$\begin{array}{l}\text { Northern Territory } \\
\text { inland }\end{array}$} & \multirow[b]{2}{*}{ Total } \\
\hline & Bamaga & Injinoo & $\begin{array}{l}\text { New } \\
\text { Mapoon }\end{array}$ & Seisa & Umagico & & Katherine I & $\begin{array}{l}\text { Katherine } \\
\text { II }\end{array}$ & \\
\hline A & $7 *$ & $2 \dagger$ & 2 & 2 & 5 & 9 & & & 27 \\
\hline B & & & & $1^{*}$ & & 3 & & & 4 \\
\hline $\mathrm{C}$ & 2 & 1 & 4 & 1 & 5 & 13 & & & 26 \\
\hline $\mathrm{D}$ & & & & 2 & 2 & & 15 & 11 & 30 \\
\hline$A+B$ & 2 & 2 & 4 & 1 & 1 & 4 & & & 14 \\
\hline$A+C$ & 1 & & 1 & 1 & & 1 & & & 4 \\
\hline$B+C$ & & & 1 & 1 & $2^{\dagger}$ & 4 & & & 8 \\
\hline$A+B+C$ & 5 & 2 & 2 & 2 & 2 & 7 & & & 20 \\
\hline All 4 & & & & 3 & 1 & & & & 4 \\
\hline Total & 17 & 7 & 14 & 14 & 18 & 41 & 15 & 11 & $137 \ddagger$ \\
\hline
\end{tabular}

* One dog was first collared in Bamaga, second time in Seisia and counted twice.

$\dagger$ One dog was first collared in Injinoo, second and third time in Umagico and counted twice.

$\$$ Because of $*$ and $\dagger$, two dogs were counted twice, correct number of dogs $=135$.

$P<0 \cdot 001$ ), as previously described by Bombara et al. [32]. Established breeds different from the common 'community dog' were only found in the NPA. Information on the use of the dogs for hunting was recorded in inland Northern Territory $(4 / 26=15 \%)$ and in the NPA $(22 / 56=39 \%)$, where significantly more hunting dogs were found $\left(\chi^{2}=6.72, P=0.035\right)$. Age and sex were similarly distributed $\left(\chi^{2}=2 \cdot 27, P\right.$ $=0 \cdot 321)$ among the regions.

The core HR size $(50 \%$ isopleth of the BRB density kernel) ranged from 0.17 to 2.33 ha with a median of $0 \cdot 35$ ha (Table 3). The range of the extended HRs was $0 \cdot 86-40 \cdot 46$ ha with a median of $4 \cdot 48$ ha. These results include the HR sizes already published for the data from time point A [28], time point B and those from Katherine I and II [33] and from time point C [32].

\section{Mixed effect model results}

In the univariable model analysis, the variables community, region, season, breed and sex had $P$ values of $<0 \cdot 3$ for HR95 as the outcome variable; and in addition, hunting and reproductive status for the outcome variable HR50 (Table 4). The predictors community or region, sex and reproductive status including first-order interaction, season and breed were further integrated into multivariable analyses. Hunting was also found to have a $P$ value of $<0.3$ for the prediction of HR50, however, because of a lot of missing values $(39 \%)$ this variable was not considered in the multivariable model.
In the multivariable model of the outcome variable HR50, the model including sex and reproductive status (including their interaction) was identified as the model with only significant fixed effects. For HR95 as the outcome variable, the final model included the fixed effects season, sex and reproductive status (Table 5). Because the reproductive status was not available from all of the dogs (Table 2), the final model included 200 instead of 215 observations (sets of GPS data). The variables age, region and community were tested as confounders. None of these variables were identified as confounders for the HR95 model, but region and community were for the HR50 model. The model including the variable region had a lower AIC (263) than the one including community $(\mathrm{AIC}=275)$. Also, region is biologically more plausible to cause confounding because neutering of dogs was found to be significantly more common in Galiwin'ku than in the other regions.

The effect of the dog's sex on HR size, both core (HR50) and extended (HR95), significantly depended on whether the dogs were neutered or entire (Table 5, Fig. 2). Entire males were found to have a significantly larger core HR than neutered males (Table 5). The same trend was observed for the extended HR $(P=0 \cdot 10$, Table 5). Neutered females tended to have a larger extended HR (HR95) than entire females $(P=0.085)$, which was not observed for the core HR $(P=0 \cdot 69)$. Finally, the extended HR was estimated to be larger in the pre- than in the post-wet season (Table 5). 
Table 2. Structure of the study population of domestic dogs used in a GPS collar study in three regions in Northern Australia between September 2013 and June 2015; these variables were tested as predictors of roaming (home range)

\begin{tabular}{ll}
\hline \hline Variable & $n(\%)$ \\
\hline Sex & 135 \\
Male & $77(57)$ \\
Female & $58(43)$ \\
Reproductive status & 122 \\
Entire & $57(47)$ \\
Neutered & $65(53)$ \\
Age & 135 \\
Young & $39(29)$ \\
Adult & $82(61)$ \\
Old & $14(10)$ \\
Breed & 135 \\
Community dog & $105(78)$ \\
Small breed & $2(1)$ \\
Middle size breed & $17(13)$ \\
Large breed & $11(8)$ \\
Used as hunting dog & 83 \\
Yes & $27(33)$ \\
No & $56(67)$ \\
Percentage of dingo DNA & 56 \\
& Range $0 \%-32 \%$, \\
I & IQR $1 \%-4 \% *$ \\
Hybrids (dogs-dingo) & Median $2 \%$ \\
Yes & 56 \\
No & $12(21)$ \\
Pre-wet & $44(79)$ \\
Post-wet & 135 \\
\hline \hline & $95(70)$ \\
Season & $40(30)$ \\
\hline
\end{tabular}

* Inter-quartile range.

Table 3. Home range sizes in hectares of 135 free-roaming dogs in Northern Australia calculated from 215 GPS datasets collected between September 2013 and June 2015

\begin{tabular}{llllllr}
\hline \hline & $2.5 \%$ & $25 \%$ & $50 \%$ & Mean & $75 \%$ & $97 \cdot 5 \%$ \\
\hline HR50* & 0.19 & 0.29 & 0.35 & 0.45 & 0.52 & 1.04 \\
HR95* & 1.42 & 3.29 & 4.48 & 6.79 & 8.18 & 25.50 \\
\hline \hline
\end{tabular}

Dogs were collared between once and four times over a period of 2 years.

* HR50 and HR95: size of the 50\% and 95\% isopleth, respectively, of the density kernel using the biased random bridge method.

The HR sizes were right skewed and therefore were log transformed, to achieve normality (Fig. S1). The distributions were homoscedastic (Fig. S1).

\section{DISCUSSION}

We estimated the HR size of 135 FRDD in eight Aboriginal and Torres Strait Islander communities in three regions in Northern Australia, both coastal and inland; and explored the influence of intrinsic (sex, reproductive status, age, breed, genetics and usage for hunting) and extrinsic (community, region and season) factors on roaming activity (HR size). The estimated HR sizes and shapes reflect what we have found in earlier studies [28, 32]: the dogs spent most of their time around their owner's house and the majority of dogs did not roam further than 250 $\mathrm{m}$ in diameter; however, a small number of dogs roamed more extensively. Comparable HR sizes have also been observed in other studies of FRDD in Australia [27] and globally [25, 29, 37]. In some studies larger sizes have been reported $[23,30]$. The methods used in each of these studies need to be considered when comparing HR sizes estimated for roaming dogs from different studies.

The main focus of our study was on identifying potential predictors of roaming, as measured by HR. Male dogs had both larger core and extended HR sizes than females in the case of entire dogs; this difference was not observed for neutered dogs. Also, entire males roamed further than neutered males for both HR levels, although for the extended HR it was only a trend. This may have implications for the transmission of infectious diseases. Non-castrated males were identified as the group with the largest HR sizes, which would lead to higher contact rates and thus disease spread over larger areas. Neutered females were identified to be the group with the second largest HR, although only for the extended HR size and only as a trend. The same result has been found in other studies investigating the roaming behaviour of FRDD. Sparkes et al. monitored 17 dogs with GPS collars in Wurrumiyanga, an Aboriginal community on Tiwi Island in Northern Australia; they also identified males to roam further [23]. Also, Gunata observed a significantly larger HR size in five male compared with five female dogs on the island of Bali, Indonesia [25]. In a study in Tibet, male dogs were found to travel longer distances from the owner's house than females [30]. Conversely, studies have also been published in which differences in HR size between the sexes of FRDDs could not be identified. For example, in a study in Kyrgyzstan in which 26 males and seven females were included, no difference in HR size was detected [29]. These findings might 
Table 4. Coefficient, standard error and overall $P$ values of the univariable mixed effect regression models with the logarithmic core (HR50) and extended home range (HR95) as outcome variables

\begin{tabular}{|c|c|c|c|c|c|c|}
\hline \multirow[b]{2}{*}{ Variable (reference level) } & \multicolumn{3}{|c|}{ Core home range (HR50) } & \multicolumn{3}{|c|}{ Extended home range (HR95) } \\
\hline & Coeff & S.E. & $P$ value & Coeff & S.E. & $P$ value \\
\hline \multicolumn{7}{|l|}{ Community } \\
\hline Bamaga & Ref lev* & & $0 \cdot 114$ & Ref lev & & $0 \cdot 005$ \\
\hline Seisia & $-0 \cdot 08$ & $0 \cdot 14$ & & $-0 \cdot 17$ & $0 \cdot 21$ & \\
\hline Injinoo & $0 \cdot 08$ & $0 \cdot 18$ & & $-0 \cdot 22$ & $0 \cdot 26$ & \\
\hline New Mapoon & $-0 \cdot 13$ & $0 \cdot 15$ & & $-0 \cdot 41$ & $0 \cdot 22$ & \\
\hline Umagico & $0 \cdot 14$ & $0 \cdot 14$ & & $0 \cdot 10$ & $0 \cdot 21$ & \\
\hline Galiwin'ku & -0.09 & $0 \cdot 12$ & & $-0 \cdot 23$ & $0 \cdot 18$ & \\
\hline Katherine I & $-0 \cdot 36$ & $0 \cdot 15$ & & $-0 \cdot 91$ & $0 \cdot 23$ & \\
\hline Katherine II & $-0 \cdot 05$ & $0 \cdot 17$ & & $-0 \cdot 21$ & $0 \cdot 26$ & \\
\hline \multicolumn{7}{|l|}{ Region } \\
\hline Elcho & Ref lev & & 0.087 & Ref lev & & $0 \cdot 009$ \\
\hline Katherine & $-0 \cdot 14$ & $0 \cdot 11$ & & $-0 \cdot 39$ & $0 \cdot 17$ & \\
\hline NPA & 0.09 & $0 \cdot 08$ & & $0 \cdot 11$ & $0 \cdot 13$ & \\
\hline \multicolumn{7}{|l|}{ Season } \\
\hline Post-wet & Ref lev & & 0.036 & Ref lev & & $<0.001$ \\
\hline Pre-wet & $0 \cdot 11$ & $0 \cdot 05$ & & $0 \cdot 39$ & $0 \cdot 09$ & \\
\hline \multicolumn{7}{|l|}{ Sex } \\
\hline Female & Ref lev & & $0 \cdot 151$ & Ref lev & & $0 \cdot 271$ \\
\hline Male & $0 \cdot 11$ & 0.08 & & $0 \cdot 13$ & $0 \cdot 11$ & \\
\hline \multicolumn{7}{|l|}{ Reproductive status } \\
\hline Entire & Ref lev & & $0 \cdot 149$ & Ref lev & & $0 \cdot 651$ \\
\hline Neutered & $-0 \cdot 11$ & $0 \cdot 08$ & & $-0 \cdot 05$ & $0 \cdot 12$ & \\
\hline \multicolumn{7}{|l|}{ Age } \\
\hline Adult & Ref lev & & $0 \cdot 543$ & Ref lev & & $0 \cdot 874$ \\
\hline Young & $0 \cdot 09$ & $0 \cdot 09$ & & $0 \cdot 07$ & $0 \cdot 14$ & \\
\hline Old & $-0 \cdot 3$ & $0 \cdot 12$ & & $0 \cdot 02$ & $0 \cdot 18$ & \\
\hline \multicolumn{7}{|l|}{ Hunting } \\
\hline No & Ref lev & & $0 \cdot 223$ & Ref lev & & $0 \cdot 310$ \\
\hline Yes & $0 \cdot 12$ & $0 \cdot 10$ & & $0 \cdot 14$ & $0 \cdot 14$ & \\
\hline \multicolumn{7}{|l|}{ Breed } \\
\hline Camp dog & Ref lev & & $0 \cdot 103$ & Ref lev & & $0 \cdot 079$ \\
\hline Large & $0 \cdot 35$ & $0 \cdot 14$ & & $0 \cdot 58$ & $0 \cdot 22$ & \\
\hline Middle size & $0 \cdot 07$ & $0 \cdot 11$ & & $0 \cdot 03$ & $0 \cdot 16$ & \\
\hline Small & $-0 \cdot 04$ & $0 \cdot 28$ & & $0 \cdot 12$ & $0 \cdot 43$ & \\
\hline \multicolumn{7}{|l|}{ Hybrid } \\
\hline No & Ref lev & & $0 \cdot 706$ & Ref lev & & $0 \cdot 858$ \\
\hline Yes & $0 \cdot 05$ & $0 \cdot 14$ & & $-0 \cdot 04$ & $0 \cdot 20$ & \\
\hline$\%$ dingo DNA & $<0.01$ & $0 \cdot 01$ & $0 \cdot 651$ & $<-0.01$ & $0 \cdot 01$ & 0.686 \\
\hline
\end{tabular}

Variables with bold $P$ values $(<0 \cdot 3)$ were further explored in multivariable models.

* Reference level.

be caused by inadequate sample size. The current study is likely the largest of its type undertaken, and benefits from much greater statistical power.

We observed that dogs had a larger extended HR size in the pre- than the post-wet season, but no difference was found for the core HR. One explanation might be the physical nature of the wet (monsoonal) season in this environment: large areas of standing water could present a barrier to roaming. Another may be the abundance of water diminishes the need for animals to travel in search of resources. In addition, following rains there is increased food availability (both for herbivores and carnivores) and this further reduces HR size. How these factors, which are of higher relevance for wildlife, influence roaming of FRDDs is unknown. Typically, domestic dogs like those observed in our study communities have sufficient water resources around houses, even in the dry 
Table 5. Regression coefficient values and their standard errors (logarithmic scale), $P$ values and number of observations per level of the fixed effects included in the final mixed effect regression models for the two outcome variables HR50 (core home range) and HR95 (extended home range); regression coefficients are additionally presented in back-transform values ( $e^{\text {coefficient }}$ )

\begin{tabular}{|c|c|c|c|c|}
\hline Fixed effects with levels & Coefficient $\left(e^{\text {coefficient }}\right)$ & S.E. & $P$ value & $n$ \\
\hline \multicolumn{5}{|c|}{ Outcome variable $=\ln (\mathrm{HR} 50)^{*}$} \\
\hline \multicolumn{5}{|l|}{ Sex } \\
\hline Male & Reference level & & & 108 \\
\hline Female & $-0 \cdot 26(0 \cdot 77)$ & $0 \cdot 12$ & $0 \cdot 04$ & 92 \\
\hline \multicolumn{5}{|l|}{ Reproductive status } \\
\hline Entire & Reference level & & & 89 \\
\hline Neutered & $-0 \cdot 24(0 \cdot 79)$ & $0 \cdot 12$ & 0.05 & 111 \\
\hline \multicolumn{5}{|c|}{ Interaction term sex $\times$ reproductive status } \\
\hline Male, entire & Reference level & & & 62 \\
\hline Female, neutered & $0 \cdot 31(1 \cdot 36)$ & $0 \cdot 16$ & 0.06 & 65 \\
\hline \multicolumn{5}{|c|}{ Outcome variable $=\ln ($ HR95 $) \dagger$} \\
\hline \multicolumn{5}{|l|}{ Sex } \\
\hline Male & Reference level & & & 108 \\
\hline Female & $-0 \cdot 40(0 \cdot 67)$ & $0 \cdot 18$ & 0.03 & 92 \\
\hline \multicolumn{5}{|l|}{ Reproductive status } \\
\hline Entire & Reference level & & & 89 \\
\hline Neutered & $-0 \cdot 26(0 \cdot 77)$ & $0 \cdot 15$ & $0 \cdot 10$ & 111 \\
\hline \multicolumn{5}{|c|}{ Interaction term sex $\times$ reproductive status } \\
\hline Male, entire & Reference level & & & 62 \\
\hline Female, neutered & $0 \cdot 56(1.75)$ & $0 \cdot 23$ & $0 \cdot 018$ & 65 \\
\hline \multicolumn{5}{|l|}{ Season } \\
\hline Post-wet & Reference level & & & 82 \\
\hline Pre-wet & $0 \cdot 35(1 \cdot 42)$ & $0 \cdot 09$ & $<0 \cdot 01$ & 118 \\
\hline
\end{tabular}

Dog identification was included as a random effect.

$*$ Intra-class correlation coefficient (ICC) 0·48; Akaike's Information Criterion (AIC) $=263$.

$\dagger$ ICC 0.33; $\mathrm{AIC}=435$.

season. Although FRDDs might not be regularly fed, even occasional feeding would be expected to reduce impacts of season. Finally, breeding might be a cause for the influence of season on roaming. Regardless of the reason, our finding of a difference in roaming pre- vs. post-wet season would impact on the spread of disease within FRDD: contacts over larger areas will be more likely during the dry season and therefore disease spread might occur more rapidly and over larger areas at this time of the year.

Age was not identified as a significant predictor of HR size. We enrolled dogs in this study from 7 months to 12 years of age (estimated based on information supplied by owners). However, most of the owners were not sure about the age of their dogs and we therefore categorised age as young, adult and old. Misclassification bias cannot be excluded, but this is unlikely because the same, non-significant association was found when age was tested as two categories (young $v s$. adult). The same potential problem of misclassification might have occurred for the variable breed (also not significantly associated with HR size). Breed was classified based on the dogs' appearance and specific, established breeds (compared with the typical 'camp dog' appearance seen in most Indigenous communities) were only found in one of the three regions (the NPA). Consequently, small sample sizes and an unbalanced distribution of the data made it difficult to investigate breed as a predictor of roaming size. A more objective variable included was dog genetics, specifically the proportion of dingo genetic background. However, because genetic samples were only collected during one visit in two of the regions, data on this variable were only available for $41 \%$ of the dogs enrolled in this study (Table 2). Again, the small sample size and unbalanced distribution of the data made investigation of this variable difficult. Finally, whether or not dogs were used for hunting also was not found to be significantly associated with roaming. Again, this information was not available for all dogs enrolled (Table 2) and also was not equally distributed between 

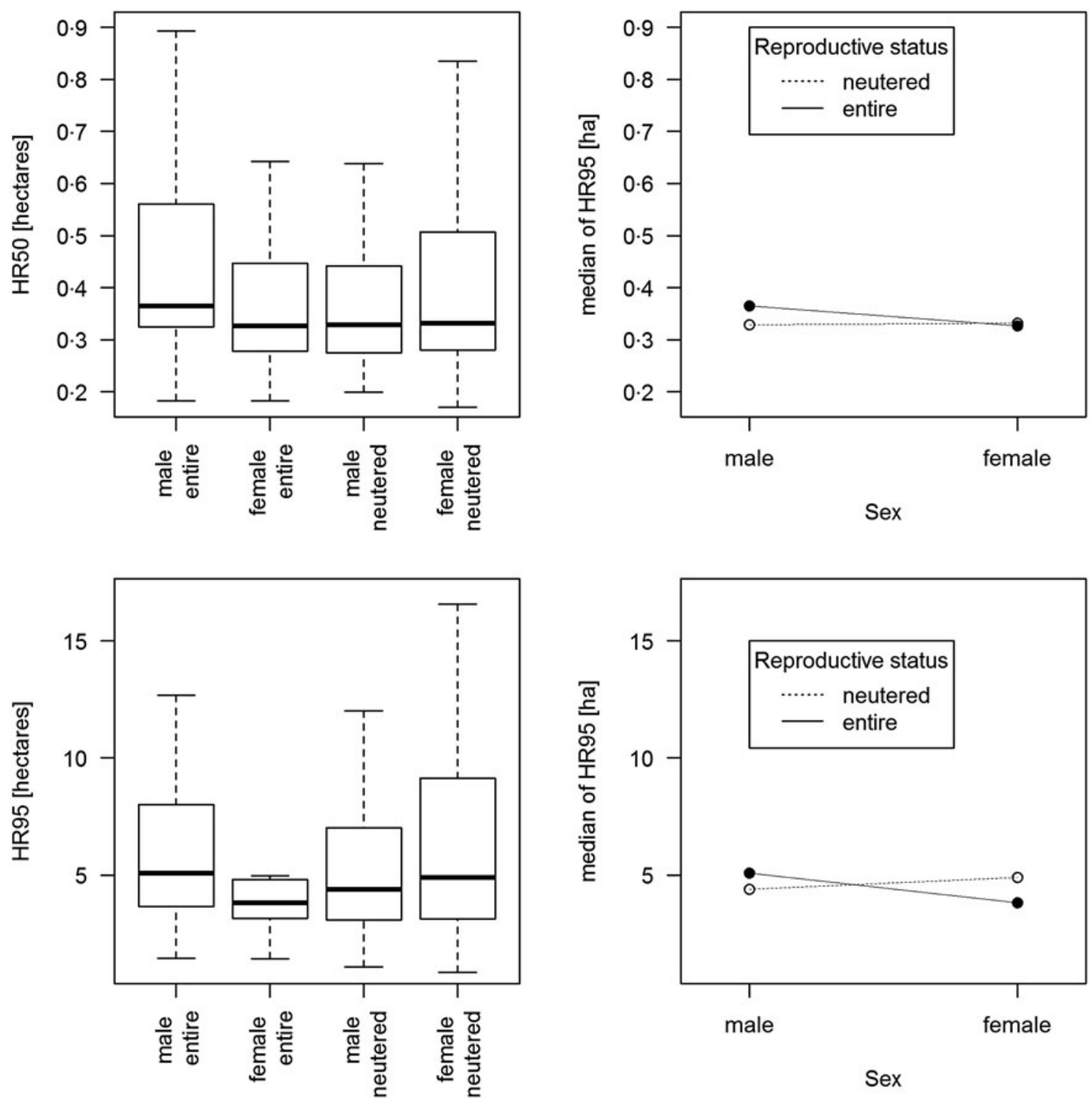

Fig. 2. Boxplots and interaction plots of the core (HR50, top line) and extended (HR95, bottom) home ranges of 200 observations from $135 \mathrm{dogs}$ from Aboriginal communities in Northern Australia, depending on their sex and reproductive status. Outliers are not shown. Data were collected between September 2013 and June 2015.

the three study regions. Vaniscotte et al. found that the tethering of dogs during the day did not influence the distance they roamed during the night [30]. A similar situation might have occurred in the current study with respect to hunting: some of the hunting dogs were chained up during the day, but some were chained up (or otherwise confined) all the time except during hunting activities.

There are a range of other predictors that might influence the roaming of FRDD that were not included in the model fit to the data. First, the dog population density was only tested indirectly via the variable 'community'. The range in community densities was high (57-415 dogs $\left./ \mathrm{km}^{2}\right)$, and during screening this variable was found to be significantly associated with extended HR size (Table 4). However, it was not identified as a significant predictor in the multivariable analysis, although a trend was observed $(P=0.0890)$. Further simple correlation analysis between HR size and community density did not confirm an association (Spearman correlation coefficient 0.076 $(P=0.267)$ and 0.084 $(P=0.220)$ for HR50 and HR95, respectively) (Fig. 3). Second, the female dog reproductive cycle might influence the roaming behaviour of both males and females. When questioning dog owners, animal management 

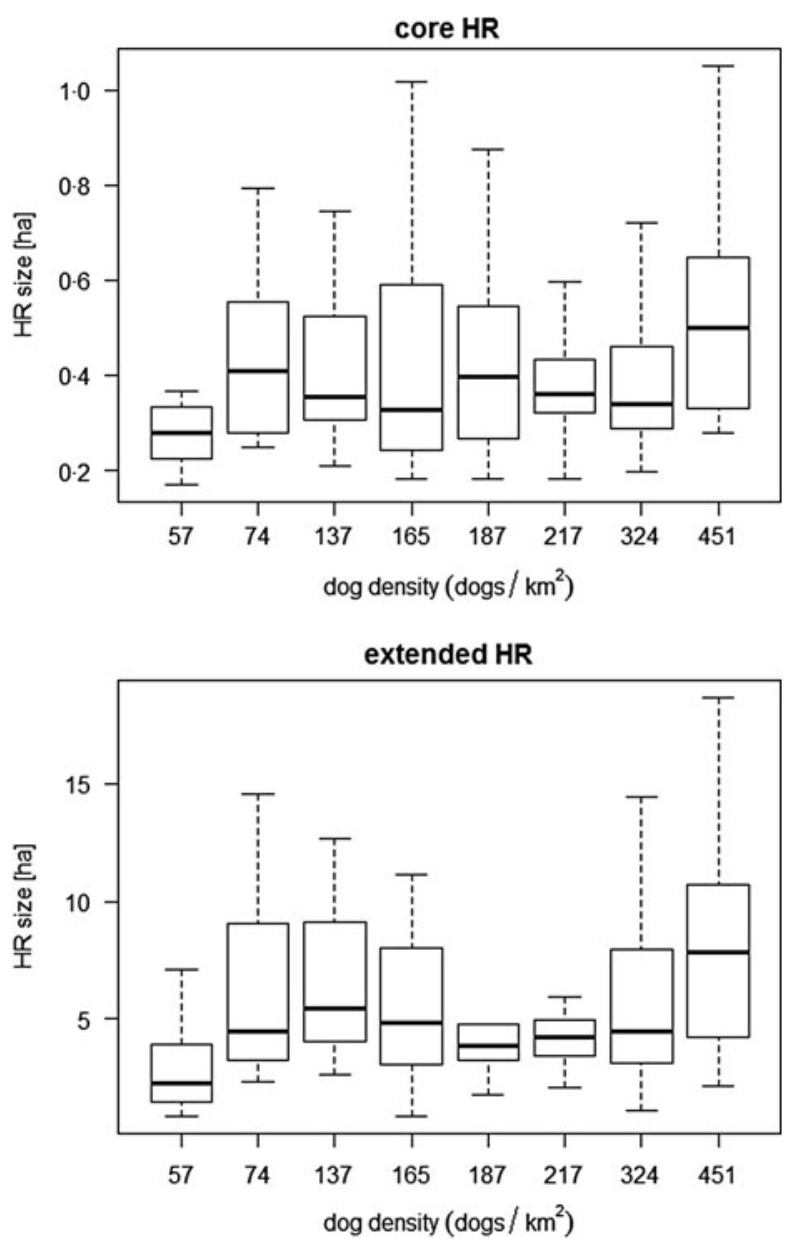

Fig. 3. Boxplot of home range sizes compared with the dog density within the community of data collection. The core and extended HR size represented the 50\% and $95 \%$ isopleth level, respectively, of a total of 200 observations in 135 FRDD in Northern Australia.

workers and veterinarians practicing in these communities, it was not possible to identify a specific season when female dogs are in oestrus. Assuming that community dogs breed throughout the year, it can be expected that, on average for the dog population, the influence of this predictor on roaming is low.

There are potentially additional predictors not investigated here. Among these are factors associated with human behaviour and dog management practices, of which only the use of dogs for hunting was investigated. It can be expected that such factors are predictors of the roaming behaviour of FRDD. First, availability of food resources has been found to influence the HR size of wild dogs and other canids. For example, HR size comparison between resource-rich and less rich regions was demonstrated to be different in fox populations in Australia [38].
Meek also discussed non-human associated hunting as a critical parameter for HR size, which is expected to be associated with the feeding practices of the dogs [27]. Whether the feeding practices of FRDD populations living within communities influence their HR size is something that needs investigation using fieldcollected data. With respect to disease transmission, it would be crucial to know whether dogs that were not fed roamed further and might therefore pose a greater risk for spread disease over a larger area. Second, we observed that several dogs accompanied their owners on daily activities such as to work, to the store or for visits (data not presented). Investigation on the correlation between the dog and owner activities would allow us to conclude how independent is the roaming of FRDD. If such an association is found to be strong, a strategy for controlling infectious disease spread would be to prevent dogs from accompanying their owners, but such a measure would be difficult to implement.

Individual dogs had relatively constant HR sizes within our dataset, which was indicated by the high intra-class coefficients $(0.33$ and $0 \cdot 47$, Table 5$)$. Also, we did not observe a significant difference between the HR sizes of individual dogs at different time points within the same season (Wilcoxon signed rank test, $P>0 \cdot 125$ ). For the 19 dogs in the NPA with available data from time point $\mathrm{A}$ and $\mathrm{C}$, the number of data collection days at time point $\mathrm{A}$ was 1-4 (median 3) whereas for time point $C$ it was 5-14 days (median 7). This provides some evidence that even a short data collection period of 2-3 days adequately represents the dogs roaming behaviour, leading to acceptable internal validity of the study. Nevertheless, long-term monitoring of domestic dogs is warranted to investigate suitable monitoring periods, an area of current active research at the same study site. External validity i.e. how much the results presented here can be extrapolated to other FRDD populations, is more critical. By including several communities and regions - both coastal and inland, a variable that was not identified as being significant for HR size - our findings are likely valid for other remote Indigenous communities in Australia. However, external validity for FRDD populations globally would need further investigation in dog populations in different environments.

We conclude that sex and the reproductive status are predictors of HR size of FRDD and that uncastrated males are the largest roamers. This should be considered for planning control strategies of infectious 
diseases, especially for measures (such as vaccination) that target individual animals. Further investigation of additional potential predictors - such as feeding practices or dog density - as well as similar studies in other FRDD populations globally will complete our knowledge on what drives free roaming domestic dogs to roam.

\section{SUPPLEMENTARY MATERIAL}

The supplementary material for this article can be found at https://doi.org/10.1017/S095026881700022X

\section{ACKNOWLEDGEMENTS}

This work was supported by the Wildlife and Exotic Disease Preparedness Program of the Australian Department of Agriculture (funding periods 2012-13 and 2013-14) and the Swiss National Science Foundation (S.D., grant number PASMP3-142737). The authors thank numerous people who were involved in the data collection within the Aboriginal and Torres Strait Islander communities: Frank Mau, George Mara and Ron Wapau from the NPA Regional Office; Philippa Dhagapan, Virginia Barratj, Sharon Wunungmurra, Sacha Woodburn and Emma Kennedy from the East Arnhem Regional Office; Alex Burleigh from the Aboriginal Community Veterinary Services, Katherine and Peter Fleming from Vertebrate Pest Research Unit, Biosecurity NSW. They also thank Peter Thomson (the University of Sydney) for advice in statistics with $\mathrm{R}$.

\section{DECLARATION OF INTERESTS}

None.

\section{REFERENCES}

1. Vila C, et al. Multiple and ancient origins of the domestic dog. Science 1997; 276: 1687-1689.

2. Slater MR. The role of veterinary epidemiology in the study of free-roaming dogs and cats. Preventive Veterinary Medicine 2001; 48: 273-286.

3. Young JK, et al. Is wildlife going to the dogs? Impacts of feral and free-roaming dogs on wildlife populations. Bioscience 2011; 61: 125-132.

4. Lembo $\mathbf{T}$, et al. Zoonoses Prevention, Control, and Elimination in Dogs. Dogs, Zoonoses and Public Health. Wallingford: CABI, 2013, pp. 205-258.

5. Jenkins D, et al. Echinococcus granulosus and other intestinal helminths: current status of prevalence and management in rural dogs of eastern Australia. Australian Veterinary Journal 2014; 92: 292-298.

6. Bradburyl L, Corlette S. Dog health program in Numbulwar, a remote Aboriginal community in east Arnhem Land. Australian Veterinary Journal 2006; 84: 317-320.

7. Meloni B, et al. The prevalence of giardia and other intestinal parasites in children, dogs and cats from aboriginal communities in the Kimberley. Medical Journal of Australia 1993; 158: 157-159.

8. Walton SF, et al. Genetically distinct dog-derived and human-derived Sarcoptes scabiei in scabies-endemic communities in Northern Australia. American Journal of Tropical Medicine and Hygiene 1999; 61: 542-547.

9. Gaskin S, et al. The zoonotic potential of dogs in aboriginal communities in central Australia. Environmental Health 2007; 7: 36-45.

10. Brown G, et al. Detection of Anaplasma platys and Babesia canis vogeli and their impact on platelet numbers in free-roaming dogs associated with remote aboriginal communities in Australia. Australian Veterinary Journal 2006; 84: 321-325.

11. Hii $\mathbf{S}$, et al. Canine vector-borne disease pathogens in dogs from south-east Queensland and north-east Northern Territory. Australian Veterinary Journal 2012; 90: 130-135.

12. Barker EN, et al. Haemoparasites of free-roaming dogs associated with several remote Aboriginal communities in Australia. BMC Veterinary Research 2012; 8: 55.

13. Murray CJL, et al. Global, regional, and national agesex specific all-cause and cause-specific mortality for 240 causes of death, 1990-2013: a systematic analysis for the Global Burden of Disease Study 2013. Lancet 2015; 385: $117-171$.

14. Hampson K, et al. Estimating the global burden of endemic canine rabies. PLoS Neglected Tropical Diseases 2015; 9: e0003709.

15. Conan A, et al. Population dynamics of owned, freeroaming dogs: implications for rabies control. PLoS Neglected Tropical Diseases 2015; 9: e0004177.

16. Gsell AS, et al. Domestic dog demographic structure and dynamics relevant to rabies control planning in urban areas in Africa: the case of Iringa, Tanzania. BMC Veterinary Research 2012; 8: 236.

17. Tenzin $\mathbf{T}$, et al. Free-roaming dog population estimation and status of the dog population management and rabies control program in Dhaka City, Bangladesh. PLoS Neglected Tropical Diseases 2015; 9: e0003784.

18. Mustiana A, et al. Owned and unowned dog population estimation, dog management and dog bites to inform rabies prevention and response on Lombok Island, Indonesia. PLOS ONE 2015; 10: e0124092.

19. Morters MK, et al. The demography of free-roaming dog populations and applications to disease and population control. Journal of Applied Ecology 2014; 51: 1096-1106.

20. Dalla Villa P, et al. Free-roaming dog control among OIE-member countries. Preventive Veterinary Medicine 2010; 97: 58-63. 
21. Mindekem R, et al. Impact of canine demography on rabies transmission in N'djamena, Chad. Médecine tropicale: revue du Corps de santeé colonial 2005; 65: 53-58.

22. El Berbri I, et al. Knowledge, attitudes and practices with regard to the presence, transmission, impact, and control of cystic echinococcosis in Sidi Kacem Province, Morocco. Infectious Diseases of Poverty 2015; 4: 48.

23. Sparkes J, et al. Effects of sex and reproductive state on interactions between free-roaming domestic dogs. PLoS ONE 2014; 9: e116053.

24. Dias RA, et al. Size and spatial distribution of stray dog population in the University of São Paulo campus, Brazil. Preventive Veterinary Medicine 2013; 110: 263-273.

25. Gunata IK. The Bio-Ecology of Dogs and Implementation of Radiotelemetry Technology to Determine Dog Home Ranges in Conjunction with Rabies Vaccination Programs in the District of Badung, Bali. Indonesia: Udayana University Bali, 2011.

26. Beck A. The Ecology of Stray Dogs: A Study of FreeRanging Urban Animals [Internet]. West Lafayette, Indiana: Purdue University Press, 2002, 117 p.

27. Meek $\mathbf{P}$. The movement, roaming behaviour and home range of free-roaming domestic dogs, Canis lupus familiaris, in coastal New South Wales. Wildlife Research 1999; 26: 847-855.

28. Dürr S, Ward MP. Roaming behaviour and home range estimation of domestic dogs in Aboriginal and Torres Strait Islander communities in Northern Australia using four different methods. Preventive Veterinary Medicine 2014; 117: 340-357.

29. Van Kesteren F, et al. Dog ownership, dog behaviour and transmission of Echinococcus spp. in the Alay Valley, southern Kyrgyzstan. Parasitology 2013; 140: $1674-1684$
30. Vaniscotte A, et al. Role of dog behaviour and environmental fecal contamination in transmission of Echinococcus multilocularis in Tibetan communities. Parasitology 2011; 138: $1316-1329$.

31. Stephens D, et al. Death by sex in an Australian icon: a continent-wide survey reveals extensive hybridization between dingoes and domestic dogs. Molecular Ecology 2015; 24: 5643-5656.

32. Bombara C, et al. Roaming of dogs in remote Indigenous communities in Northern Australia and potential interaction between community and wild dogs. Australian Veterinary Journal 2017; in press.

33. Molloy S, et al. Roaming behaviour of dogs in four remote Aboriginal communities in the Northern Territory, Australia: preliminary investigations. Australian Veterinary Journal 2017; in press. doi: 10.1111/avj.12562.

34. Burleigh A, McMahon S, Kiely S. Owned dog and cat populations in remote Indigenous communities in the Northern Territory: a retrospective study. Australian Veterinary Journal 2015; 93: 145-150.

35. Forin-Wiart MA, et al. Performance and accuracy of lightweight and low-cost GPS data loggers according to antenna positions, fix intervals, habitats and animal movements. PLoS ONE 2015; 10: e0129271.

36. Benhamou S. Dynamic approach to space and habitat use based on biased random bridges. PLOS ONE 2011; 6: e14592.

37. Berman M, Dunbar I. The social behaviour of freeranging suburban dogs. Applied Animal Ethology 1983; 10: 5-17.

38. Saunders G, et al. Urban foxes (vulpes vulpes): Food acquisition, time and energy budgeting of a generalized predator. Zoological Society of London Symposia. 1993; 65: 215-234. 